\title{
Hereditary breast and ovarian cancer: risk assessment in minority women and provider knowledge gaps
}

Nancy M Paris, MS, FACHE, ${ }^{a}$ Sheryl GA Gabram-Mendola, MD, MBA, FACS, ${ }^{\mathrm{b}}$ Alice S Kerber, MN, APRN, ACNS-BC, AOCN, APNG, , Jean O'Connor, JD, MPH, DrPH, Barbara E Crane, MN, APRN, ${ }^{c}$ Monique L Martin, MPH, CHES, ${ }^{\mathrm{d}}$ Lucy Brannon Traxler, MD, ${ }^{\mathrm{e}}$ Roland Matthews, MD, ${ }_{\mathrm{f}}$ Christopher Parker MBBS, MPH, ${ }^{\mathrm{g}}$ Rachel Webster, MMSc, CGC, ${ }^{\text {h }}$ Elizabeth Schmitt, MMSc, CGC, ${ }^{\mathrm{i}}$ Dana Meaney-Delman, MD, MPH, Navya Nair, MD, MPH, ${ }^{\mathrm{k}}$ Victoria L Green, MD, JD, MBA, ${ }^{1}$ and Cecelia A Bellcross, PhD, MS, $\mathrm{CGC}^{\mathrm{m}}$

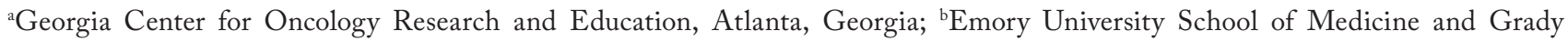
Memorial Hospital, Atlanta, Georgia; 'Office of Prevention, Screening and Treatment, Georgia Department of Public Health, Atlanta, Georgia; ${ }^{d}$ Deloitte, Atlanta, Georgia; ${ }^{\mathrm{e}}$ Mary Black Health System, Spartanburg, South Carolina; ${ }^{\mathrm{f}}$ Morehouse School of Medicine and Georgia Cancer Center for Excellence at Grady, Atlanta, Georgia; ${ }^{g}$ Georgia Health Policy Center, Andrew Young School of Policy Studies, Georgia State University, Atlanta, Georgia; hTh University of Texas, MD Anderson Cancer Center,

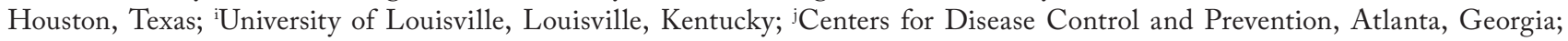
${ }^{k}$ Icahn School of Medicine, Mount Sinai Hospital, New York; ${ }^{1}$ Georgia Cancer Center for Excellence at Grady, Atlanta, Georgia; and ${ }^{m}$ Department of Human Genetics, Emory University School of Medicine, Atlanta, Georgia

Background The Georgia Breast Cancer Genomics Project identified minority and underserved women at high risk for hereditary breast and ovarian cancer based on family history. Education, web-based screening, genetic counseling, and testing were provided in public health and primary care settings in accordance with evidence-based recommendations and guidelines.

Objectives To assess risk of hereditary breast and ovarian cancer (HBOC) among minority and underserved women, provide genetic services according to evidence-based guidelines, and evaluate provider knowledge of HBOC.

Methods The Georgia Department of Public Health established this project through a cooperative agreement with the Centers for Disease Control and Prevention. HBOC screening and genetic services were provided in 13 public health centers and federally qualified health centers. Staff received training on genetics and risk assessment using the Breast Cancer Genetics Referral Screening Tool (B-RST). Providers and medical residents were surveyed on their knowledge of HBOC. Young women with breast cancer were surveyed on receipt of genetic services.

Results More than 5,400 women were successfully screened; 79\% identified as racial/ethnic minorities. 5\% of women screened positive on the B-RST, which is consistent with HBOC prevalence; $79 \%$ agreed to follow up. $23 \%$ met criteria for increased risk of $B R C A 1 / 2$ mutation and received genetic counseling and testing. Surveys revealed profound gaps in knowledge among physicians and medical residents and lack of delivery of evidence-based genetic services to survivors.

Conclusions The genomics project demonstrated the efficacy of population-based screening to identify high-risk women before they receive a diagnosis of cancer. A high percentage of women who screened positive also completed genetic counseling and testing. Access to the benefits of HBOC management to prevent cancer and decrease mortality among minority and underserved women depends on improvements in knowledge of genetics and evidence-based practice by providers.

Funding/sponsorship This project was funded through a cooperative agreement from the Centers for Disease Control and Prevention to the Georgia Department of Public Health from 201 1-2014.

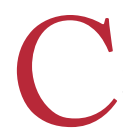
ancers are collectively the leading causes of premature death in the United States and their incidence is increasing as the population ages. ${ }^{1}$ Breast cancer is the most common of the cancer diagnoses and the second leading cause of cancer death in women in both the US and in the state of Georgia. ${ }^{2,3}$ Ovarian cancer is the fifth leading cause of cancer death and is often diagnosed at more advanced stages. Although multiple factors have been associated with an increased risk of breast and ovarian cancer, family history remains the most significant and consistent predictor of cancer risk. ${ }^{4,5}$ Systematic collection of family history, referral of high-risk women to cancer genetic services, and selective genetic testing can identify a mutation in a hereditary cancer gene. When followed by cascade 
testing of at-risk relatives for the known familial mutation, the benefits of appropriate management are extended beyond the individual. The goal of identifying those at risk for hereditary cancer is to reduce morbidity and mortality through early diagnosis and effective prevention strategies. ${ }^{6}$

Although hereditary breast and ovarian cancer (HBOC) syndrome associated with mutations in the BRCA $1 / 2$ genes is not common in the general population, women who carry mutations in those genes face a substantially higher risk of breast and ovarian cancer than the general population. Data from the National Cancer Institute (NCI) have revealed that between 1 in 400 and 1 in 800 women in the general population have a $B R C A 1 / 2$ mutation. ${ }^{7}$ About 1 in 40 women of Ashkenazi Jewish descent carry a founder BRCA $1 / 2$ mutation. ${ }^{7}$ The average population lifetime risk for breast cancer is $1: 8(13 \%)$ and for ovarian cancer, 1:70 (1.5\%); lifetime risks for breast and ovarian cancer in $B R C A 1 / 2$ mutation carriers range from $40 \%-80 \%$ and from $10 \%-40 \%$, respectively. ${ }^{8}$

Offering genetic counseling and testing for $B R C A 1 / 2$ to women who are at increased risk for HBOC can guide targeted cancer surveillance, prevention strategies, and influence lifestyle decisions that reduce cancer-related morbidity and mortality. ${ }^{7}$ For women who test positive for a $B R C A$ $1 / 2$ mutation, interventions that include chemoprevention, enhanced surveillance, or prophylactic mastectomy and salpingo-oophorectomy can decrease the incidence of breast and ovarian cancer by up to $95 \%$ and $80 \%$, respectively. ${ }^{9-13}$ Despite these clear benefits, the literature demonstrates under-referral to and under-use of genetic counseling and testing, particularly for minority and underserved women. Racial/ethnic minorities include African Americans, Hispanics, and Asians. There were no Native Americans to report. Underserved is defined as those seeking care in free or reduced-cost public health centers and federally qualified health centers. ${ }^{14,15}$

The Precision Medicine Initiative launched by President Obama in January 2015 is accelerating research on the methods by which we prevent, diagnose, and treat cancers, and has emphasized the potential impact of analyzing inherited genetic variations. ${ }^{16}$ Concurrently, changes in federal law have resulted in the wider incorporation of population health improvement concepts into public health practice and patient-centered medical homes. Population health improvement relies on the provision of evidence-based, multidisciplinary care that emphasizes care coordination, integration of screening into practice, and addressing health disparities to prevent illness and provide better quality care. ${ }^{17}$

Evidence-based guidelines of the US Preventive Services Task Force (USPSTF), the National Comprehensive
Cancer Network (NCCN) and the American College of Surgeons' Commission on Cancer $(\mathrm{CoC})$ indicate that women with a personal or family history of breast and/or ovarian cancer may benefit from genetic counseling and testing. Family history indicators associated with increased likelihood of potentially harmful $B R C A 1 / 2$ mutations include a known $B R C A$ 1/2 mutation in the family; breast cancer diagnosis before age 50; ovarian cancer at any age; bilateral breast cancer; triple-negative breast cancer; presence of breast and ovarian cancer in the same individual; presence of breast cancer in male family members; multiple cases of breast cancer in the family; 1 or more family members with 2 primary types of $B R C A$ 1/2-related cancer; and Ashkenazi Jewish ancestry. ${ }^{18-20}$

Use of genetic services and extension of the benefits of genetic research to minority and underserved women is a specific objective of Healthy People 2020, an initiative by the Office of Disease Prevention and Health Promotion, though it is still in its infancy. Minority and underserved women face substantial obstacles that limit the benefits of cancer genetic risk assessment, testing, and cancer risk management. These women may lack information regarding the impact of family history on cancer risk; have limited geographic and financial access to cancer risk assessment; and receive fewer referrals for appropriate services. ${ }^{21,22}$ Increasing the proportion of minority and underserved women with a family history of breast and/or ovarian cancer who receive genetic counseling and testing should reduce cancer disparities, enhance cancer and noncancer health outcomes, and ensure that these women are receiving evidence-based, high-quality care. ${ }^{23-25}$

\section{Georgia Breast Cancer Genomics project}

The cancer burden experienced by Georgians is complicated by geographic barriers, socioeconomic status, and access to care. Georgia's population is 10.2 million and its land mass makes it the largest state east of the Mississippi river. Twenty-five percent of the population is rural; 19\% lives below the poverty level, and $18 \%$ is uninsured. Racial and ethnic minorities comprise $45 \%$ of the population, including 32\% African Americans, 9\% Hispanics, and 4\% Asians. ${ }^{8,3}$ The early identification of minority and underserved women with $B R C A$ 1/2 mutations requires integration of education, risk assessment, and genetic services into settings where these women seek health care. To be most effective, health care providers must engender the trust of women in their care. ${ }^{14,15,23}$

The Georgia Breast Cancer Genomics project was designed to address disparities in access to care in patients in Georgia who are at risk for hereditary cancer. The spe-

Accepted for publication April 1 1, 2016. Correspondence: Nancy M Paris, MS, FACHE; nparis@georgiacore.org. Disclosures: The authors report no disclosures or conflicts of interest. JCSO 2016;14:261-267. @2016 Frontline Medical Communications. doi: 10.12788/ jcso.0215. 
cific goal was to identify premenopausal, minority, and underserved women (aged 18-49 years) who were at high risk for HBOC before a diagnosis of cancer. The Chronic Disease Section of the Georgia Department of Public Health (DPH) established the genomics project in 2011 through a cooperative agreement with the Centers for Disease Control and Prevention (CDC). Key partners included the Georgia Center for Oncology Research and Education (Georgia CORE), Emory University School of Medicine, Georgia State University, and Morehouse School of Medicine. Each of those partners was integral to the success of the project, providing guidance on the genomics project to the DPH through 2014. Data collected as part of the project goals during 2011-2014 are referenced herein. At the successful conclusion of the cooperative agreement, Georgia DPH incorporated the genomics project into the Statewide Breast and Cervical Cancer Program.

The genomics project used population health improvement strategies to identify minority and underserved women who were at high risk for HBOC through the following activities:

- Educating public health professionals and primary care providers ( $\mathrm{PCPs}$ ) on cancer genetics and methods to identify women at increased risk for $\mathrm{HBOC}$ using a multidisciplinary genomics curriculum;

- Implementing validated web-based screening for women at risk for $\mathrm{HBOC}$ in settings serving minority and underserved women, including public health centers and federally qualified health centers;

- Delivering HBOC education, risk assessment, and resources to minority and underserved women in community-based public health and primary care settings;

- Providing genetic counseling and comprehensive cancer risk assessment to women identified as at-risk for HBOC by screening;

- Facilitating genetic testing for those meeting NCCN and other guidelines.

\section{Method}

In the first 3 years of operation, the genomics project integrated screening for women at risk for HBOC, as well as provision of genetic services, into 13 community-based public health and primary care settings in which free and reduced cost care was provided. No additional clinic staff was required to implement the program: members of the project provided administrative, educational, and clinical support. Potential financial barriers to access to the support were eliminated by providing genetic counseling and testing free of charge through a combination of grant funding and industry support.

Screening for women at risk for HBOC was conducted by staff trained by multidisciplinary members of the genomics project to implement the Breast Cancer Genetics Referral Screening Tool (B-RST). The B-RST is a validated screening tool designed to identify women who should be referred for cancer genetic services for HBOC. The tool is available at www.BreastCancerGeneScreen.org in English and Spanish. ${ }^{26-28}$ In 2013, B-RST was recommended by the USPSTF as a simple, quick, and clinically useful tool to help identify women who might benefit from a genetic counseling referral. The screening tool is comprised of 6 questions, takes less than 5 minutes to complete, and uses select family history information to identify women at risk for $\mathrm{HBOC} .{ }^{20}$ Based on a clinical algorithm, the tool provides 3 possible results related to the risk of developing HBOC: negative-low risk, negative-moderate risk, or positive-high risk. HIPAA-compliant electronic data capture was integrated into a secured database located on the www.BreastCancerGeneScreen.org website to facilitate the collection of patient demographic data, track risk assessment results, and disseminate HBOC-related information and resources. Women with positive-high risk screening results were offered formal genetic counseling services, including detailed family history documentation and cancer risk assessment by a qualified genetics professional in person or by telephone. ${ }^{29}$

\section{Results}

More than 5,400 women were successfully screened using the B-RST during 2011-2014. In all, 4,293 (79\%) of those women self-identified as racial or ethnic minorities. Women who screened positive/high risk on the B-RST represented 5\% (287) of the total number screened, which aligns with the HBOC prevalence rate across the country. Of the 227 (79\%) women who screened positive and agreed to follow-up, 173 (67\%) were successfully contacted. During a genetic counseling session, a detailed family history was collected, and 40 women (23\%) met criteria for an increased risk for a BRCA 1/2 mutation. These women underwent in-depth genetic counseling and all chose to proceed with genetic testing. Among the 40 women who were tested for $B R C A$ 1/2, 1 pathogenic mutation and 4 variants of uncertain significance were identified. All of the women received education and risk-appropriate guidance for screening and healthy lifestyle behaviors. Nine women were diagnosed with breast cancer following a positive/ high risk B-RST screening; none of them were found to have a $B R C A 1 / 2$ mutation, though all had a suggestive family history. ${ }^{29}$

\section{Surveys of PCPs, medical residents, and breast cancer survivors}

Pilot surveys of primary care providers, medical residents, and young breast cancer survivors were designed and conducted by the genomics project staff to inform development and implementation of HBOC education, risk assessment, and genetic services. Two of the surveys assessed clinician knowledge of HBOC genetics - the 
first among PCPs, and the second among primary care resident physicians in training. Accurate understanding of genetics and HBOC among clinicians is essential to provision of evidence-based care including the appropriate referral of at-risk patients to genetic services. This knowledge is also critical for the future of precision medicine. A third survey was designed to assess the use of genetic services and testing among young breast cancer survivors (diagnosed under age 50 years) who met NCCN guidelines for further genetic risk evaluation. ${ }^{19}$

\section{Knowledge of $\mathrm{HBOC}$ syndrome among Georgia PCPs (2012)}

A 34-question survey was developed to assess basic knowledge of HBOC and application of genetic principles among primary care providers. ${ }^{30}$ One thousand surveys were mailed to randomly selected licensed health care providers (family practice, internal medicine, midlevel provider, and obstetrics and gynecology) in Georgia, with a $\$ 5$ cash incentive and postage-paid envelope. Surveys were completed by 369 (37\%) respondents with 275 (75\%) of those eligible for analysis. Among the providers, about 35\% rated themselves as highly confident in their knowledge of HBOC. They demonstrated some knowledge of basic genetic principles, but were unable to consistently identify individuals at greatest risk for HBOC. Knowledge deficits included limited understanding of inheritance patterns and failure to recognize the risk impact of ovarian cancer (96\%) or a known BRCA1/2 mutation in the family (74\%). A summary of these results is shown in Table 1 , which includes analysis of the percentage of correct answers by specialty. Ob-gyns consistently scored higher than the other specialties, though this difference was significant only for the questions regarding inheritance pattern and risk given a familial mutation. Of note is the finding that only $3.9 \%$ of respondents identified a woman with ovarian cancer and a family history of ovarian cancer as high risk.

The results of the surveys support further educational efforts with a specialty and case-based approach directed toward primary health care providers as critical to ensuring that patients at greatest risk for $\mathrm{HBOC}$ are identified and referred for genetic counseling. ${ }^{30}$

\section{HBOC education needs of Georgia primary care residency training programs (2014)}

Surveys were distributed electronically to 22 primary care medical residency training programs in Georgia, including family medicine, obstetrics and gynecology, and internal medicine specialties, and responses were received from 10 facilities (45\% response rate). Program directors or faculty representatives were asked to complete a survey on HBOC education within their curricula. Residents currently enrolled in the medical residency programs $(n=$ 122) were asked to complete a knowledge survey of key HBOC concepts. Fifty-seven percent of respondents did not recall having received education regarding $\mathrm{HBOC}$; $51 \%$ were not able to identify autosomal dominant inheritance; and $31 \%$ failed to recognize that a $B R C A 1 / 2$ mutation can be maternally or paternally inherited. Self-rated confidence levels of residents were largely in the average range as presented in the Figure. Residency curricula content is presented in Table 2 .

The results of the survey suggest that targeted educational intervention during residency training may be necessary to improve the ability of PCPs to accurately identify and appropriately refer families at increased risk for $\mathrm{HBOC}$ in order to maximize the benefits of available genetic services and cancer prevention strategies. ${ }^{31}$

\section{Receipt of genetic services among young breast cancer survivors (2014)}

A 47-item anonymous survey was completed electronically by 292 young breast cancer survivors (diagnosed at younger than 50 years) through the Georgia-based breast cancer survivor support group listserv. Questions focused on referral for and receipt of cancer genetic services, with

TABLE 1 Knowledge base of primary care providers - total number of responses and percentage of correct responses

\begin{tabular}{|c|c|c|c|c|c|c|}
\hline Concept & $\begin{array}{c}\text { Total } \\
(\mathbf{N}=\mathbf{2 7 5})\end{array}$ & $\begin{array}{l}\text { Ob-gyn } \\
(n=74)\end{array}$ & $\begin{array}{l}\text { Internal } \\
\text { medicine } \\
(n=89)\end{array}$ & $\begin{array}{l}\text { Family } \\
\text { practice } \\
(n=51)\end{array}$ & $\begin{array}{l}\text { Midlevel } \\
\text { provider } \\
(n=61)\end{array}$ & $P$ value $^{a}$ \\
\hline$B R C A$ biparental inheritance pattern & $53.4 \%$ & $64.3 \%$ & $60.8 \%$ & $45.1 \%$ & $45.6 \%$ & 0.04 \\
\hline $\begin{array}{l}B R C A \text { mutation risk in first-degree rela- } \\
\text { tive of a known carrier }\end{array}$ & $39 \%$ & $54.4 \%$ & $35.3 \%$ & $37.8 \%$ & $25.9 \%$ & 0.01 \\
\hline $\begin{array}{l}\text { BRCA mutation risk in a healthy woman } \\
\text { with a family history of breast cancer }\end{array}$ & $37.1 \%$ & $42.3 \%$ & $47.1 \%$ & $30.1 \%$ & $32.8 \%$ & 0.16 \\
\hline
\end{tabular}

${ }^{\circ}$ Chi-square test. 
additional questions related to genetic services barriers, facilitators, and motivators. Forty-nine percent of respondents reported receiving a referral or recommendation for genetic counseling, with $37 \%$ actually receiving those services and $16.5 \%$ undergoing genetic testing. Twenty-seven percent reported insurance coverage as the main barrier to seeking genetic services (lack of coverage or high outof-pocket cost). Only $20 \%$ of those who were having testing reported they did so because they understood it was important to their care. Of the respondents, 181 (62\%) identified themselves as racial or ethnic minorities. These data suggest that young breast cancer survivors in Georgia are unlikely to receive genetic services in accordance with NCCN guidelines and highlight the need to incorporate genetic education and referral into routine clinical care for this group of breast cancer patients. In addition, education of young breast cancer patients will empower them to request genetic services. Policy makers should also consider innovative ways to improve referral to, uptake and receipt of cancer genetic services among young breast cancer survivors. ${ }^{32}$

\section{Summary and conclusions}

The genomics project identified minority and underserved women at high risk for HBOC (based on family history) who would not otherwise have had access to genetic/ genomic services, and it provided education, counseling, and testing in accordance with the evidence-based recommendations and guidelines. Population-based screening is recommended based on the marked increase in the lifetime risk of breast and ovarian cancer for $B R C A 1 / 2$ mutation carriers. Thus far, in Georgia, more than 5,400 minority and underserved women have undergone screening for $\mathrm{HBOC}$ as part of the genomics project. Although 1 woman was identified as having a pathogenic mutation and 4 were identified has having variants of uncertain significance, these numbers are consistent with national prevalence of HBOC. The project's impact through genetics education of providers and increased information and awareness among women is significant and is critical in light of the increased cancer burden, late stage of diagnosis, and high mortality rates in this population. As concluded by Traxler and colleagues, the "expansion of this protocol may contribute to a long-term reduction in health inequity related to cancer incidence and mortality among high-risk and minority populations." ${ }^{29}$

The population health improvement strategy described in this project - integration of screening for individuals at risk for hereditary cancer into existing clinical practice - demonstrates how a simple, practical, low-resource intervention can address existing health disparities. The introduction of web-based screening using the B-RST efficiently and effectively facilitated risk assessment for HBOC. Financial barriers were minimized by provid-

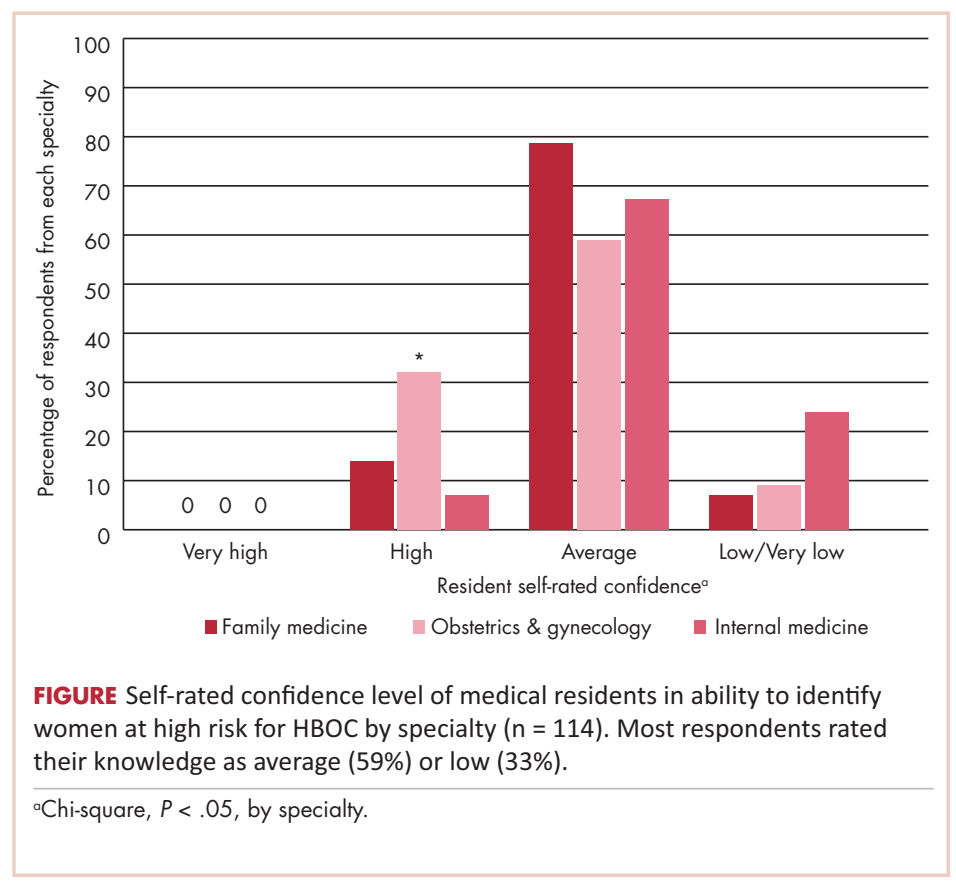

TABLE 2 HBOC residency curricula content as reported by program directors and faculty representatives

\begin{tabular}{lc} 
Curricula content & $\begin{array}{c}\text { \% included in } \\
\text { curricula }\end{array}$ \\
\hline Documentation of family history & 87.5 \\
BRCA I/2 genetics & 25 \\
HBOC red flags & 62 \\
HBOC screening and management & 50 \\
recommendations & 25 \\
\hline Identification of high-risk patients &
\end{tabular}

ing genetic services, including genetic testing, at no cost to the women served. The genomics project also demonstrated a method of incorporating cancer genetic risk assessment and services into public health centers and community-based health care settings that can be replicated and further expanded through telemedicine. A critical factor to consider in replicating this methodology is that screening for HBOC should be accompanied by education, and when appropriate, genetic counseling and testing. In addition, women who test positive for mutations should be referred to providers to discuss options to decrease future risks of cancer including chemoprevention, enhanced surveillance, and surgery.

Despite the positive results of the project, survey results revealed profound gaps in knowledge of genetics and HBOC among both PCPs and medical residents. Both groups indicated interest in educational opportunities, to enhance their knowledge of HBOC and appropriate referral practices for genetic services. Respondents' preferences 
included an interactive case-based approach with application to practice and self-guided web or journal programs with continuing education credit. Survey results also identified deficiencies in the receipt of genetic services among young women with breast cancer who are most likely to have HBOC. Access to the benefits of genetic services and HBOC management to prevent cancer and decrease mortality among minority and underserved women depends on improvements in knowledge of genetics and evidencebased practice by providers. Such improvement will also shape the future implementation of the national precision medicine initiative.

Based on our findings, we conclude that it is feasible to incorporate genetic risk assessment for HBOC into clinical practice even among diverse populations in underserved settings, and that the delivery of evidence-based care requires improved provider knowledge of genetics, $\mathrm{HBOC}$, and recognition of the importance of genetic services. Identification of $\mathrm{HBOC}$ is merely one step toward reversing cancer disparities for minority and underserved women; they must also receive appropriate genetic services, clinical management, and continuing access to low- or nocost screenings through programs such as the Breast and Cervical Cancer Program of the Georgia Department of Public Health. The genomics project highlighted the abil- ity to provide such services in a large and diverse population. Until provider awareness of HBOC referral and management guidelines is consistent with current evidence, a model of intervention such as the genomics project may be essential to population-based HBOC risk assessment. Minority and underserved women are unlikely to receive the proven and recommended benefits of evidence-based care that could prevent future cancers and reduce cancer related mortality in the absence of enhanced provider knowledge of genetics and HBOC. Future work should thus focus on development of educational interventions customized to improve provider knowledge according to specialty, with the goal of increasing the number of women at risk for $\mathrm{HBOC}$ who are appropriately referred for cancer genetic services.

\section{Acknowledgments}

The authors acknowledge the significant contributions of the following: District Health Directors and nursing staff of the Georgia Department of Public Health, in particular the counties of Bibb, Chatham, Clayton, Cobb, Columbia, Columbus, DeKalb, Gwinnett, and Thomas; the Center for Black Women's Wellness in Atlanta, Georgia; Primary Care of Southwest Georgia in Thomasville and Blakely, Georgia; Dr Kimberly Redding and Joyce Slade, RN, for their support of the initiation and continuing success of the project. And finally, special thanks to women who were willing to share their time, family histories, and personal stories so we could all learn from each other.

\section{References}

1. Collins, FS and Varmus, H. Perspective: a new initiative on precision medicine. New Engl J Med. 2015;372:793-795.

2. American Cancer Society. Cancer facts and figures 2016. http://www. cancer.org/research/cancerfactsstatistics/cancerfactsfigures2016/. Accessed April 26, 2016.

3. Georgia Department of Public Health. 2013 Georgia cancer program and data summary. http://dph.georgia.gov/sites/dph.georgia. gov/files/2013Cancer_Program_and_Data_Summary.pdf . Released August 2013. Accessed August14, 2015.

4. Cannistra SA. Cancer of the ovary. N Engl J Med. 2004;351:2519-2529.

5. Collaborative Group on Hormonal Factors in Breast Cancer. Familial breast cancer: collaborative reanalysis of individual data from 52 epidemiological studies including 58,209 women with breast cancer and 101,986 women without the disease. Lancet. 2001;358:1389-1399.

6. Centers for Disease Control and Prevention. Public health genomics. http://www.cdc.gov/genomics/famhistory/index.htm. Last updated October 30, 2015. Accessed August 14, 2015.

7. National Cancer Institute. Genetics of breast and gynecologic cancers - health professional version. http://www.cancer.gov/types/ breast/hp/breast-ovarian-genetics-pdq\#link/2723_toc. Updated March 4, 2016. Accessed August 6, 2015.

8. Chen S, Parmigiani G. Meta-analysis of BRCA 1 and BRCA 2 penetrance. J Clin Oncol. 2007;25:1329-1333.

9. De Felice F, Marchetti C, Musella A, et al. Bilateral risk-reduction mastectomy in BRCA1 and BRCA2 mutation carriers: a meta-analysis. Ann Surg Oncol. 2015;22:2876-2880.

10. Metcalfe K, Lynch HT, Foulkes WD, et al. Effect of oophorectomy on survival after breast cancer in BRCA1 and BRCA2 mutation carriers. JAMA Oncol. 2015;1:306-313.

11. Finch A, Beiner M, Lubinski J, et al; Hereditary Ovarian Cancer Clinical Study Group. Salpingo-oophorectomy and the risk of ovarian, fallopian tube, and peritoneal cancers in women with a BRCA1 or BRCA2 mutation. JAMA. 2006;296:185-192.

12. Pruthi S, Heisey RE, Bevers TB. Chemoprevention for breast cancer.

Ann Surg Oncol. 2015;22:3230-3235.

13. Xu L, Zhao Y, Chen Z, Wang Y, Chen L, Wang S. Tamoxifen and risk of contralateral breast cancer among women with inherited mutations in BRCA1 and BRCA2: a meta-analysis. Breast Cancer. 2015;22:327-334.

14. Glenn BA, Chawla N, Bastani R. Barriers to genetic testing for breast cancer risk among ethnic minority women: an exploratory study. Ethn Dis. 2012;22:267-273.

15. Hall MJ, Olapade, OI. Disparities in genetic testing: thinking outside the BRCA box. J Clin Oncol. 2006;24:2197-2203.

16. National Institutes of Health. Precision medicine initiative cohort program - building a research foundation for 21 st century medicine Precision Medicine Initiative (PMI) Working Group Report to the Advisory Committee to the Director. http://www.nih.gov/ precisionmedicine/09172015-pmi-working-group-report.pdf. Released September 17, 2015. Accessed September 30, 2015.

17. Rosenbaum S. The Patient Protection and Affordable Care Act: implications for public health policy and practice. Public Health Rep. 2011;126:130-135.

18. [American College of Surgeons] Commission on Cancer. Cancer program standards 2012: ensuring patient-centered care. https:// www.facs.org/ /media/files/quality\%20 programs/cancer/coc/programstandards2012.ashx . Published 2012. Accessed May 12, 2015.

19. Daly MB, Pilarski R, Axilbund JE, et al. Genetic/familial high risk assessment: breast and ovarian: version 2.2015. http://www.jnccn. org/content/14/2/153.full. Published 2015. Accessed August 6, 2015.

20. Moyer VA. Risk assessment, genetic counseling, and genetic testing for BRCA-related cancer in women: US Preventive Services Task Force recommendation statement. Ann Int Med. 2014;160:271-281.

21. Institute of Medicine (IOM). Genomics-enabled learning health care systems: Gathering and using genomic information to improve patient care and research: Workshop summary. http://books.nap.edu/ openbook.php?record_id=21707. Released 2015. Accessed May 17, 2015.

22. Office of Disease Prevention and Health Promotion. Healthy People 
2020. Genomics. https://www.healthypeople.gov/2020/topics-objectives/topic/genomics/objectives. Revised 2013. Accessed August 6, 2015.

23. Sheppard VB, Mays D, La Veist T, Tercyak KP. Medical mistrust influences black women's level of engagement in BRCA $1 / 2$ genetic counseling and testing. J Natl Med Assoc. 2013;105:17-22.

24. Thompson HS, Sussner K, Schwartz MD, Edwards T, Forman A Jandorf $\mathrm{L}$, et al. Receipt of genetic counseling recommendations among black women at high risk for BRCA mutations. Genet Test Mol Biomarkers. 2012;16:1257-1262.

25. American Cancer Society. Cancer facts \& figures for African Americans 2016-2018. http://www.cancer.org/acs/groups/content/@ editorial/documents/document/acspc-047403.pdf. Accessed April 26, 2016

26. Bellcross CA. Further development and evaluation of a breast/ovarian cancer genetics referral screening tool. Genet Med. 2010;12:240.

27. Bellcross CA, Kolor K, Goddard KA, Coates RJ, Reyes M, Khoury MJ. Awareness and utilization of BRCA 1/2 testing among US primary care physicians. Am J Prev Med. 2011;40:61-66.

28. Bellcross CA, Lemke AA, Pape LS, Tess AL, Meisner LT. Evaluation of a breast/ovarian cancer genetics referral screening tool in a mammography population. Genet Med. 2009;11:783-789.

29. Traxler BL, Martin ML, Kerber AS, et al. Implementing a screening tool for identifying patients at risk for hereditary breast and ovarian cancer: a statewide initiative. Ann Surg Oncol. 2014;21:3342-3347.

30. Nair N, Bellcross CA, Meaney-Delman D. Georgia primary care providers' knowledge of hereditary breast and ovarian cancer syndrome [published online ahead of print December 2015]. J Canc Educ.

31. Schmitt E, Martin M, Meaney-Delman D, Bellcross CA. hereditary breast and ovarian cancer education needs of Georgia primary care residency training programs. Poster presented at: National Society of Genetic Counselors 33rd Annual Education Conference; September 17-20, 2014; New Orleans, LA.

32. Webster R, Zhang T, Martin M, Bellcross CA. Receipt of cancer genetic services among young breast cancer survivors in Georgia. Poster presented at: National Society of Genetic Counselors 33rd Annual Education Conference; September 17-20, 2014; New Orleans, LA. 